\title{
Automatic Patient Pose Estimation Using Pressure Sensing Mattresses
}

\author{
Robert Grimm ${ }^{1,2}$, Johann Sukkau ${ }^{3}$, Joachim Hornegger ${ }^{1}$, Günther Greiner ${ }^{2}$ \\ ${ }^{1}$ Pattern Recognition Lab, University of Erlangen-Nuremberg \\ ${ }^{2}$ Chair for Computer Graphics, University of Erlangen-Nuremberg \\ ${ }^{3}$ Siemens AG, Healthcare MR, Erlangen, Germany \\ robert.grimm@informatik.uni-erlangen.de
}

\begin{abstract}
We present a system to automatically estimate the body pose of a reclined patient, based on measurement data from a pressure sensing mattress. It can be used to replace or reduce manual input in clinical imaging procedures and thus improve the workflow. The proposed method consists of two stages. First, the body posture is classified into prone, supine, and left and light lateral orientation by a k-nearestneighbor classifier. In the second algorithmic stage, a modified optimization scheme based on Powell's direction set method fits a model of the human body to the observed pressure distribution. Thus, the position of important body landmarks is estimated. For our database of 143 measurements from 16 subjects, a mean classification rate of $96.0 \%$ was achieved for the posture, and an average localization error of $6.95 \mathrm{~cm}$ for the body parts.
\end{abstract}

\section{Introduction}

Detailed knowledge about a patient's position on an examination table is very valuable information in many clinical workflows. For example, prior to a Magnetic Resonance Imaging (MRI) examination, the radiologist has to define the center of the examination region on the patient by means of a laser crosshair. Moreover, the fundamental alignment of the patient has to be entered into the system, e.g. that he is in left lateral posture with the feet towards the scanner bore. In this example, the desired localization information are the orientation (feet first) and the posture (left lateral). Knowledge of the pose (position and orientation of body and limbs) supersedes the laser crosshair, as it implicitly defines the location of a given body region on patient table. A similar scenario is the positioning of $\mathrm{C}$-arm Computed Tomography (CT) scanners [1]. In this paper, we show how these tedious steps of manual input can be made redundant by an automatic pose estimation system.

Approaches to markerless human pose estimation or patient localization based on optical sensors $[2,3]$ are, in general, not sufficiently robust since they tolerate only limited visual occlusion of the subject. Instead, our system relies on an array of pressure sensors that is placed between the mattress and the patient. 
Harada et al. [4] compared measurement data of a pressure sensing mattress to a database of 180 templates that were pre-computed by estimating the pressure distribution of a simple human body model. The dimensions of the model have to be adjusted manually to match those of the actual subject. The limited number of training data accounts for merely three to five different configurations of the four degrees of freedom in their model. With today's computing power, larger databases are feasible, but an exhaustive database for a detailed body model would still be too big. In later work, Harada et al. [5] described a motion tracking system based on the physical forces exerted on the mattress surface. However, this approach requires manual initialization of the pose of the body model. Seo, Oh, and Lee [6] also utilized an array of pressure sensors to discriminate between supine, left, right, and sitting posture of a human reclining on a bed. Without specifying the size of the tested data set, the authors quote an accuracy of $93.6 \%$ for classification with a radial basis function neural network.

Unlike previous approaches, our system is fully automatic. It can reliably classify the fundamental posture with an accuracy of $96.0 \%$, even in the challenging case where the knees are not bent in lateral positions. Since the sensors are placed underneath the patient, our method is not impaired if the patient is covered by a blanket.

The detailed body pose is analyzed using a custom synthetic body model with 15 degrees of freedom that allows arbitrary joint angles.

\section{Materials and Methods}

Arrays of pressure sensors are available from several vendors and in a variety of sizes. A popular use case is ergonomic optimization, e.g. for prostheses, seats, or mattresses. Typically, they are used for long-term monitoring, but, as they can be read out at a rate of $10 \mathrm{~Hz}$ or more, also real-time measurements are possible. At each node with index $(i, j)$ of the array, a sensor measures the applied physical force $\mathrm{F}(i, j) \in \mathbb{R}_{0}^{+}$. The pressure sensors can be calibrated to quantify the force in metric units.

On the algorithmic side, our system is composed of two stages that first classify the posture and then estimate the articulated body pose.

\subsection{Posture Classification}

Most pressure is applied to the mattress by the shoulders and hips. By localizing these regions of maximal pressure relative to the whole body, the patient orientation (head first/feet first) is determined. To further distinguish between prone, supine, and left and right lateral postures, a k-nearest-neighbor $(\mathrm{kNN})$ classification compares the measured pressure distribution to a set of labeled training data. The measurement is assigned the same class as the majority of the $k$ most similar training data belong to. The similarity between two measurements is computed as the sum-of-squares distance when both data are aligned such that their center of mass coincides. 


\subsection{Model-Based Pose Estimation}

In the second stage, we use a 3D human body model composed from elliptic cylinders and ellipsoids to generate a synthetic pressure distribution. In the pose estimation process, the configuration of this model is iteratively adjusted to maximize the similarity between the generated pressure distribution and the measurement data. The model is augmented by additional geometry to heuristically simulate points of particularly high forces on the surface, as shown in Figure 1(a). For example, in supine posture, the weight of the legs rests mostly on the heels. By contrast, the knees are usually clearly visible in prone posture.

For each posture, the 15 degrees of freedom in the model configuration are described by a vector $\boldsymbol{\theta}=(\boldsymbol{t}, \phi, s, \boldsymbol{\delta}) \in \mathbb{R}^{15}$. It defines the global translation $t \in \mathbb{R}^{2}$ and a global rotation $\phi$ in the plane parallel to the sensor mattress, the scale factor $s$ determining the body height of the model, and a vector of Euler angles $\boldsymbol{\delta} \in \mathbb{R}^{11}$ that indicate the joint angles in the plane parallel to the mattress surface. A synthetic pressure distribution $\hat{\mathrm{F}}(\boldsymbol{\theta}, i, j)$ is generated by sampling a depth map of the model at coordinate $(i, j)$ using OpenGL. In an iterative process, the model configuration vector $\hat{\boldsymbol{\theta}}$ is estimated that minimizes the sum-of-squares difference between the generated and the observed data:

$$
\hat{\boldsymbol{\theta}}=\arg \min _{\boldsymbol{\theta}} \sum_{i, j}(\hat{\mathrm{F}}(\boldsymbol{\theta}, i, j)-\mathrm{F}(i, j))^{2}
$$

The algorithm is based on Powell's direction set method [7] that performs consecutive optimizations along linear dimensions and requires no gradient computation. Our implementation performs several cycles; in each cycle, a linear search is conducted individually for each degree of freedom. The model configurations are generated according to a fixed, heuristically determined schedule.

\section{$2.3 \quad$ Experiments}

To evaluate the accuracy of our algorithms, a total of 143 different pressure distributions was acquired from 13 male and 3 female volunteers. For every subject, at least two measurements were conducted in each of the four fundamental postures. In our experiments, we used the XSensor X3 PX100:26.64.01 mattress. Its array of $64 \times 26$ capacitive sensors provides a spatial resolution of $3.175 \mathrm{~cm}$. The sensor sheet is flexible, as thin as $1 \mathrm{~mm}$, and sized $203.2 \mathrm{~cm} \times 81.28 \mathrm{~cm}$. It was placed on top of a camping mat and a soft blanket.

The two algorithmic stages of posture classification and pose estimation were analyzed independently. For kNN classification, cross-validation was performed, using, in turns, the data of four volunteers for training and testing on the rest.

For pose estimation, the data were upsampled to a resolution of $256 \times 104$ pixels. Five cycles of the optimization procedure, corresponding to approximately 500 tested configurations, were performed for every dataset. From the estimated model pose, the metric location of important body landmarks was computed and compared to the corresponding, manually labeled gold standard coordinates. 
Table 1. Confusion matrix of kNN classification results. Rows indicate the true label.

\begin{tabular}{lllll}
\hline & Left & Right & Prone & Supine \\
\hline Left & 74 & 1 & 0 & 9 \\
Right & 1 & 87 & 0 & 2 \\
Prone & 0 & 0 & 85 & 2 \\
Supine & 2 & 0 & 0 & 166 \\
\hline
\end{tabular}

Table 2. Average Euclidean distance between estimated landmark positions and gold standard, in $\mathrm{cm}$. $\mathrm{L}$ and $\mathrm{R}$ denote the left and right joints.

\begin{tabular}{|c|c|c|c|c|c|c|c|c|}
\hline & & Right & 1 & 87 & 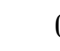 & & 2 & \\
\hline & & Prone & 0 & 0 & & 5 & 2 & \\
\hline & & Supine & 2 & 0 & ( & & 166 & \\
\hline & Crest & Neck & Shou & lder & Hip & & Kne & \\
\hline & & & $\mathrm{L}$ & $\mathrm{R}$ & $\mathrm{L}$ & $\mathrm{R}$ & $\mathrm{L}$ & $\mathrm{R}$ \\
\hline $\mathrm{eft}$ & 11.0 & 6.2 & 7.4 & 7.2 & 5.2 & 6.6 & 8.3 & - \\
\hline Right & 7.6 & 7.7 & 6.1 & 5.8 & 8.0 & 5.2 & - & 8.4 \\
\hline Prone & 14.9 & 7.0 & 12.1 & 9.8 & 7.2 & 6.1 & 5.1 & 3.1 \\
\hline Supine & 2.9 & 4.2 & 6.5 & 6.8 & 4.7 & 4.0 & 6.5 & 6.9 \\
\hline
\end{tabular}

\section{Results}

The heuristic algorithm to determine the orientation of a patient succeeded in all 143 cases. For the $\mathrm{kNN}$ classifier $(\mathrm{k}=5)$, the cumulative confusion matrix from the four cross-validation runs is shown in Table 1 . The average classification rate, ie. the percentage of correct classifications, is $96.0 \%$.

Figure 1(b) shows measurement data and, as an overlay, the skeleton of the estimated model pose. The average Euclidean distances between the estimated position of selected landmarks and the corresponding gold standard coordinates are given in Table 2. The mean error over all landmarks in is $6.95 \mathrm{~cm}$. Since in lateral positions the opposite side of the body is not captured, no errors were computed for the affected knees. The computation time for pose estimation in one dataset is about $5 \mathrm{~s}$ with a single-threaded implementation on a $2.26 \mathrm{GHz}$ Intel Core2 Duo CPU and an ATI Mobility RadeonHD 3400 GPU.

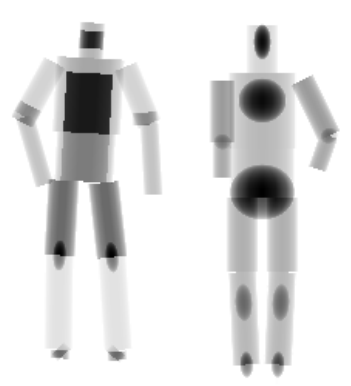

(a)

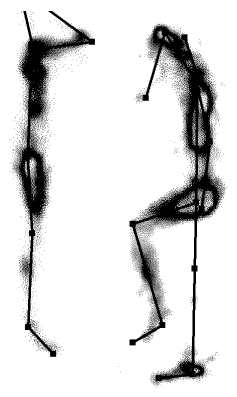

(b)

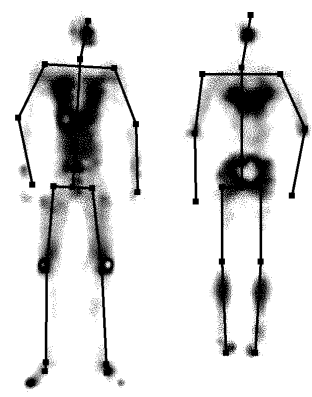

Fig. 1. (a) Models for prone and supine posture. (b) Input data (left, right, prone, supine), overlaid with model skeleton after five pose estimation cycles. 


\section{Discussion}

With the sensor mattress embedded e.g. into the patient table of a Magnetic Resonance Imaging system, the presented system automatically provides localization information about the patient. This reduces manual interactions that are required before every examination today.

The qualitative and quantitative results confirm the robustness of the proposed methods to compute the orientation and posture of a patient on a bed as well as the location of individual body parts. The reason for the lower accuracy for head and shoulders in prone position is that some volunteers placed their head on the arms, which leads to an unspecific pressure distribution in that area. By contrast, in some small regions a lot of force is concentrated, e.g. at the knees in prone posture and the head in supine posture. A mismatch of the corresponding model geometry in such areas is heavily penalized by the objective function, leading to higher accuracy.

The presented approach is limited to the localization of extremities that touch the mattress. Incorporation of an optical camera could provide complementary information from a viewpoint above the patient. Since currently only a single data acquisition is used for pose estimation, another aspect subject to future work is the extension to motion tracking over a period of time.

Acknowledgement. The authors gratefully acknowledge support for this work provided by Siemens AG, Healthcare MR, Erlangen, Germany and Siemens Mindit Magnetic Resonance Ltd., Shenzhen, P.R. China.

\section{References}

1. Schaller C, Rohkohl C, Penne J, et al. Inverse C-arm positioning for interventional procedures using real-time body part detection. Lect Notes Computer Sci. 2009; p. 549-56.

2. Moeslund TB, Hilton A, Krüger V. A survey of advances in vision-based human motion capture and analysis. Comp Vis Imag Under. 2006;104(2-3):90-126.

3. Lee MW, Nevatia R. Human pose tracking in monocular sequence using multilevel structured models. IEEE Trans Pat Anal Mach Intell. 2009;31(1):27-38.

4. Harada T, Mori T, Nishida Y, et al. Body parts positions and posture estimation system based on pressure distribution image. In: Proc IEEE Int Conf Robot Autom. vol. 2 ; 1999. p. $968-75$.

5. Harada T, Sato T, Mori T. Human motion tracking system based on skeleton and surface integration model using pressure sensors distribution bed. In: Proc Workshop on Human Motion; 2000. p. 99-106.

6. Seo KH, Oh C, Lee JJ. Intelligent bed robot system: pose estimation using sensor distribution mattress. In: Proc IEEE Int Conf Robot Biomim; 2004. p. 828-32.

7. Powell MJD. An efficient method for finding the minimum of a function of several variables without calculating derivatives. Comput J. 1964;7:155-62. 